\title{
FLEBOTOMÍNEOS (DIPTERA: PSYCHODIDAE) NO ESTADO DO MATO GROSSO, MUNICIPIO DE CHAPADA DOS GUIMARÃES, BRASIL.
}

\author{
Ceclie M.B BIANCARDI ${ }^{*}$, Eloy Guillermo CASTELLÓN*.
}

RESUMO - Estudo sobre a fauna de flebotomineos (Diptera: Psychodidae), no Municipio de Chapada dos Guimarães, Mato Grosso, Brasil, mostrou a presença de duas espécies de Brumptomyia e 43 espécies de Lutzomyia, todas registradas pela primeira vez no Estado de Mato Grosso. Durante o periodo de três anos (1993 a 1995), coletamos espécimes para conhecer a diversidade de espécies e aspectos ecológicos da fauna de flebotomíneos da área. Chama-se a atenção para a presença de $L$. ayrozai, $L$. flaviscutellata e $L$. whitmani vetores incriminados na transmissão da leishmaniose cutânea e L. longipalpis, vetor da leishmaniose visceral no Brasil.

Palavras-chaves: Diptera, Psychodidae, Flebotomineos, Mato Grosso.

Phlebotomine Sand Flies (Diptera: Psychodidae) in the Mato Grosso State, Municipality of Chapada dos Guimarães, Brazil.

ABSTRACT - Studies on the Phlebotomine sandfly fauna (Diptera:Psychodidae) of Chapada dos Guimarães, municipality of Mato Grosso State, Brazil, showed the presence of 2 species of Brumptomyia and 43 species of Lutzomyia; all were found for the first time in Mato Grosso state. During a period of three years (1993 to 1995) collections were carried out to know the species diversity and ecological aspects of the sandfly fauna in that area. The authors draw attention to the presence of L. ayrozai, L. flaviscutellata and L. whitmani, known vectors of cutaneous leishmaniasis, and, L. longipalpis, major vector of visceral leishmaniasis in Brasil.

Key-Words: Diptera, Psychodidae, Sand Flies, Mato Grosso.

\section{INTRODUÇÃO}

A leishmaniose americana é uma zoonose produzida por várias espécies de parasitas do gênero Leishmania transmitida entre mamiferos, muitas vezes silvestres, através de vetores conhecidos por flebotomineos, dipteros da familia Psychodidae, sub-familia Phlebotominae. O homen é considerado hospedeiro acidental, sem papel na cadeia de manutenção ou disseminação de parasitas na natureza (Lainson, 1983).

Fraiha et al. (1978) enfatizaram que o conhecimento da fauna flebotomínica de uma área, constitui um passo fundamental para o desenvolvimento de programas de controle das leishmanioses. Os vetores incriminados na transmissão das leishmanioses neotropicais para o homen, pertencen aos subgêneros Lutzomyia, Nyssomyia e Psychodopygus.

$\mathrm{Na}$ região da Amazônia brasileira, vários autores estudaram os aspectos biológicos, taxionômicos e ecológicos dos flebotomineos; autoridades estão citadas em Young \& Duncan (1994).

O grande número de flebotomineos por nós coletados, despertou-nos o interesse para a realização de um estudo da fauna de flebotomineos na região que

*. Pesquisador. CPCS/INPA. Caixa Postal 478 - Manaus, Am. Brasil. 69011-970 
atualmente representa um dos maiores pólos turisticos do Estado e do Pais.

O objetivo do presente trabalho foi o de conhecer a diversidade de espécies de flebotomíneos no Municipio de Chapada dos Guimares, Estado de Mato Grosso, e reconhecer as espécies antropófilas, já incriminadas como vetores de leismanioses e detectar os possiveis abrigos naturais das formas adultas.

As informações coletadas em nossos trabalhos de pesquisa servirão como subsidios à Fundação Nacional de Saúde para que sejam utilizadas no controle da nosologia já existente na região, contornando problemas sócioeconômicos ao municipio que tem grande parte de sua economia apoiada no Turismo que ali ocorre durante $o$ ano todo.

\section{Localização e Vegetação da Área}

O munícipio de Chapada dos Guimarães dista $60 \mathrm{~km}$ de Cuiabá, capital do Estado de Mato Grosso. Morfologicamente ressalta-se nessa região a presença de extensos chapadões, suavemente ondulados que, mais ao norte, dão lugar às elevações cristalinas do Maciço Mato Grossense, as quais defrontam-se com a depressão amazônica para onde encaminha a drenagem (Fundação Cândido Rondon, 1983).

A vegetação dominante é a floresta subcaducifólia tropical, que no sentido do sul e do leste, vai gradativamente, restringindo-se aos vales e ás encostas dos interfúlvios, detendo-se no topo dos chapadões, onde domina o cerrado. Na sua porção nordeste e centro-leste, aparecem campos naturais (margem do Rio Araguaia e do Rio das Mortes, seus afluentes), (Fundação Cândido Rondon, 1983).

Os principais eixos rodoviários que servem esta região são a BR-163 (São Miguel D'Oeste - Santarèm), que se articula com a BR-364 (Limeira- fronteira com o Peru) e a BR-158 (Barra dos Garças - São Fèlix do Araguaia) que se conecta com a BR-070 (Brasilia - fronteira com a Bolivia).

\section{MATERIAL E MÉTODOS}

Durante o período de Janeiro de 1993 a Dezembro de 1995, foram realizadas capturas de flebotomíneos nas áreas do Rio Manso, Colégio Buriti e Fazenda Passagem do Mamão - Vale da Benção, no Municipio de Chapada dos Guimarães no Estado de Mato Grosso.

As coletas foram realizadas durante a primeira semana de cada mês (8 dias / mês), no período de três anos consecutivos. Na captura dos flebotomineos utilizaram-se as seguintes estratégias de coletas:

a) Armadilhas Luminosas tipo CDC: foram montadas diariamente ás 17:00 h e recolhidas no dia seguinte por volta das 7:00 h. Estas armadilhas foram colocados a alturas de $1 \mathrm{~m}-5 \mathrm{~m}$ em aréas do Rio Manso, Colégio buriti e Vale da Benção. Ali encontramos arbustos que variavam de 1 á $5 \mathrm{~m}$ de altura, das espécies murici (Byrsonina $s p$.), lixeira (Curatella americana), pau terra (Qualea parviflora), 
(Qualea multiflora) e sucupira roxa (Baudickia virgilioides).

b) Armadilhas tipo Disney: Seis (6) armadilhas foram colocadas no interior das matas, e tocas de animais silvestres. Foram montadas ás 8:00 h e recolhidas por volta de 7:00 h do dia seguinte. As iscas utilizadas, foram roedores da espécie Cavia porcellus.

c) Base de Árvores: Os flebotomíneos foram capturados em tubos de ensaio pequenos $(7 \mathrm{~cm}) \mathrm{com}$ tampas e capturadores de Castro, nos horários das 8:00 ás 11:00 h.

d) Armadilhas tipo Shannon: Utilizaram-se duas armadilhas luminosas (lâmpadas de $60 \mathrm{w}$ alimentadas à bateria de $12 \mathrm{v}$ ). Os coletores utilizaram capturadores de Castro ( 3 pôr noite) e tubos de ensaio pequenos $(7 \mathrm{~cm})$ no período de 19:00 hs às 02:00 h.

e) Coletas em tocas de Animais e fendas nas rochas: as coletas foram realizadas durante 0 dia, no horário entre 08:00 h ás 16:00 $\mathrm{h}$ usando capturadores de Castro.

Os flebotomineos coletados em armadilhas Shannon, CDC, bases das árvores, tocas de animais e fendas de rochas, foram conservados em álcool $70 \%$.

Os flebótomos coletados em armadilhas Disney foram passados em solução de detergente para retirada do óleo de ricino das bandejas e colocados em ácool 70\%.

No laboratório, os espécimes foram colocados em solução de $\mathrm{KOH}$ a $10 \%$ a quente (aquecido até quase o ponto de fervura), em seguida lavados por 3 minutos em água destilada para retirada do $\mathrm{KOH}$; ácido acético por 5 minutos, novamente na água destilada, colocados no álcool $70 \%$ por 5 minutos e em seguida clarificados no fenol por 24-48 horas.

\section{Montagem em lâmina}

$\mathrm{O}$ inseto foi colocado na lâmina sob uma gota de berlese, com o auxilio de dois estiletes de pontas finas separava-se a cabeça do tórax nos exemplares machos. Nas fêmeas separava-se a cabeça do tórax, e no abdomem, os 2 últimos segmentos abdominais a fim de visualizar as espermatecas. Em seguida, uma laminula era colocada sobre o exemplar dissecado. Após um periodo de 72 horas (a uma semana) na estufa para secagem a $37^{\circ} \mathrm{C}$, colocava-se esmalte incolor em volta da laminula para sua impermeabilizaçâo.

\section{Identificação Rápida de Flebotomos}

Para a identificação dos espécimes não montados em lâminas permanentes, o procedimento foi o seguinte: após todas as passagens para clarificação, os insetos foram distribuidos em lâminas escavadas sendo que os machos e fêmeas foram colocados separadamente e identificados diretamente no microscópio.

\section{Analise Estatistica dos Dados}

Os dados foram analisados pelos testes $\mathrm{x}$, $\mathrm{t}$-student, análise de variância e correlação múltipla, utilizando-se os pacotes estatisticos 
STATGRAPH ver. 5.01 e SYSTAT ver. 5.02 .

\section{RESULTADOS}

Nas diversas estratégias de capturas utilizadas no Municipio de Chapada dos Guimarães / MT, no período de Janeiro de 1993 a Dezembro de 1995, foram coletados 19,789 espécimes de 47 espécies de flebotomíneos dos gêneros Lutzomyia e Brumptomyia; 53,62 foram de machos e $46,38 \%$ de espécimes fêmeas (Tab. 1).

A maior frequência de espécies está representada por $L$. whitmani $(10,49 \%)$, seguida por $L$. cruzi $(9,35 \%)$, L. davisi $(8,97 \%), L$. complexa $(8,25 \%)$, L. dispar(7,08\%), L. longipalpis $(6,58 \%), L$. shannoni $(6,52 \%)$, L. fischeri $(5,51 \%)$ e L. abonnenci $(4,41 \%)$. As demais espécies somaram $32,84 \%$.

Ressaltamos que L. whitmani tem sido incriminada como vetor da Leishmania braziliensis (Young \& Arias, 1991).

A espécie L. longipalpis está reconhecidamente envolvida na transmissão da leishmaniose visceral no Brasil; L. flaviscutellata como vetor de $L$. amazonensis na Amazônia brasileira (Young \& Arias, 1991) e $L$. ayrozai e $L$. davisi recentemente envolvidas na transmissão da $L$. naiffi, agente responsável pela leishmaniose cutânea, também no Amazonas (Lainson \& Shaw, 1989).

Na tabela 1, observamos que o total de machos e fêmeas não apresentaram diferença significante
$(\mathrm{P}=0.250)$ dentro desses grupos, quando analisados estatisticamente.

Na tabela 2, evidenciamos as espécies e espécimes de flebotomíneos por estratégias de coletas. As armadilhas tipo Shannon mostraram-se mais efetivas quanto ao número de espécimes e diversidade de espécies nas coletas desses insetos, seguidas pelos métodos de capturas em buracos, tocas em pedreiras, troncos de árvores, CDC e Disney.

Com a utilização dessas estratégias, observamos uma diferença significante entre esses grupos $(\mathrm{P}=0,007)$, e aplicamos então, o teste de comparação múltipla para isolar os grupos de armadilhas que diferem, e encontramos que;

1. As armadilhas tipo Shannon foram, então, mais eficientes do que as outras estratégias utilizadas;

2. As coletas em tocas de animais e fendas em pedreiras foram mais eficientes do que as coletas com armadilhas tipo Disney.

3. As coletas em troncos de árvores foram mais eficientes do que as realizadas em armadilhas tipo Disney.

Na tabela 2 demostramos os resultados das coletas nas bases das árvores. As espécies com maiores frequeências foram L.complexa (47,03\%), L. saulensis $(19,26 \%)$ e $L$. nevesi $(16,27 \%)$. Uma espécie ainda foi relacionada como Lutzomyia sp.

$\mathrm{Na}$ estratégia de coletas com armadilhas tipo Shannon (Tab. 2) foram coletadas dezenove (19) espécies: L. whitmani representou $14,44 \%$, 
seguida por $L$. cruzi com $12,88 \%, L$. davisi com $11,22 \%, L$. dispar com $9,05 \%$, L. shannoni com $8,98 \%$ e $L$. fischeri com $7.59 \%$ do total de flebótomos coletados com esta técnica.

Nas coletas realizadas com armadilhas tipo Disney, as espécies de maior frequência foram: $L$. flaviscutellata $(42,96 \%)$, L. davisi $(30,77 \%)$ e $L$. dispar $(18,95 \%) \quad$ (Tab. 2 ). Todas as espécies foram notadamente zoofilicas.

Cinco (5) espécies foram coletadas nas armadilhas tipo CDC (Tab. 2): L. acanthopharynx (com $46,02 \%$ ) representou a espécie com maior frequência de individuos nos niveis de 1 e 2 metros, seguida de $L$. punctigeniculata $(34,89 \%)$. No nivel de 3 metros, $L$. punctigeniculata foi a mais capturada $(70,10 \%)$ seguida de $L$. antunesi $(29,36 \%)$. Não houve registro de flebotomíneos nas armadilhas nos niveis de 4 e 5 metros de altura.

Analisados estatisticamente esses dados, (análise de variância e teste $\mathrm{t}$ ), verificou-se que : para a altura de 1 a 3 metros essa diferença foi significativa $(\mathrm{p}<0,05)$.

\section{Abrigos}

Vários foram os abrigos naturais observados na região de Chapada dos Guimarães. Verificamos que as espécies do gênero Brumptomyia preferem tocas de animais no solo, como tocas de roedores. Foram coletados $B$. avellari, B. brumpti e Brumptomyia sp.

A espécie L. longipalpis importante vetor de leishmaniose vis- ceral no Brasil, foi frequentemente coletada em fendas de rochas, abundantes na região. Outras espécies menos importantes do ponto de vista epidemiológico foram capturados com esta estrategia, como assinalado na Tabela 2.

Espécies como L. bourrouli, $L$. complexa, $L$. infraspinosa, $L$. saulensis e L. nevesi, assim como Lutzomya sp. (ainda não definida) foram encontradas sobre os troncos de árvores, usando-os como local de abrigo e repouso.

\section{DISCUSSÃO}

\section{Coletas em Troncos de Árvores}

No municipio de Chapada dos Guimarães foram coletadas sete espécies de flebotomíneos do gênero Lutzomyia, utilizando-se essa metodologia, representadas por $L$. complexa, $L$. saulensis, $L$, nevesi, $L$. infraspinosa, Lutzomyia sp., $L$. coutinhoi e L. bourrouli.

Christensen \& Vasques (1982) pesquisando esses biótopos, no Panamá, coletaram 33 espécies de flebotomíneos, demonstrando assim a importância dessa metodologia em levantamentos da fauna desses insetos.

Segundo os mesmos autores, esses biótopos representaram um local de repouso que fornece um micro ambiente estável, muito utilizado pelos flebotomineos durante o dia.

Coutinho \& Barretto (1941) em coletas realizadas em áreas florestais, no, estado de São Paulo, encontraram 17 espécies e observaram que a fauna 
Tabela 1. Número de espécimes, freqûencia por sexo e total das espécies de flebotomíneos coletados no Municipio Chapada dos Guimarães $\backslash \mathrm{mt}$, no periodo de Janeiro de 1993 a Dezembro de 1995.

\begin{tabular}{|c|c|c|c|c|c|c|}
\hline \multirow[b]{2}{*}{ Espécie. } & \multicolumn{2}{|c|}{ Machos. } & \multicolumn{2}{|c|}{ Fêmeas. } & \multicolumn{2}{|c|}{ Total. } \\
\hline & 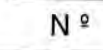 & $\%$ & $N \stackrel{0}{=}$ & $\%$ & $N=$ & $\%$ \\
\hline B. avellari & 322 & 1,62 & 0 & - & 322 & 1,63 \\
\hline B. brumpti & 11 & 0,05 & 0 & - & 11 & 0,06 \\
\hline B. sp & 0 & - & 7 & 0,03 & 07 & 0,03 \\
\hline L. abonnenci & 512 & 2,59 & 361 & 1,82 & 873 & 4,41 \\
\hline L. acanthopharynx & 337 & 1,70 & 126 & 0,63 & 463 & 2,33 \\
\hline L. antunesi & 321 & 1,63 & 97 & 0,49 & 418 & 2,11 \\
\hline L. aragoi & 206 & 1,04 & 62 & 0,31 & 268 & 1,35 \\
\hline L. ayrozai & 223 & 1,13 & 127 & 0,64 & 350 & 1,76 \\
\hline L. batyi & 4 & 0,02 & 0 & - & 04 & 0,02 \\
\hline L. barrettoi barrettoi & 7 & 0,02 & 3 & 0,01 & 10 & 0,05 \\
\hline L. bourrouli & 6 & 0,04 & 2 & 0,01 & 08 & 0,04 \\
\hline L. brasiliensis & 31 & 0,16 & 17 & 0,08 & 48 & 0,24 \\
\hline L. campbelli & 8 & 0,04 & 0 & - & 08 & 0,04 \\
\hline L. carmelinoi & 19 & 0,10 & 4 & 0,02 & 23 & 0,11 \\
\hline L. carrerai carrerai & 129 & 0,65 & 215 & 1,08 & 344 & 1,73 \\
\hline L. claustrei & 68 & 0,34 & 39 & 0,19 & 107 & 0,54 \\
\hline L. complexa & 719 & 3,63 & 915 & 4,62 & 1.633 & 8,25 \\
\hline L. coutinhoi & 15 & 0,07 & 0 & - & 15 & 0,07 \\
\hline L. cruzi & 976 & 4,93 & 875 & 4,42 & 1.851 & 9,35 \\
\hline L. davisi & 1.065 & 5,38 & 711 & 3,59 & 1.776 & 8,97 \\
\hline L. dispar & 623 & 3,14 & 779 & 3,93 & 1.402 & 7,08 \\
\hline L. evandroi & 37 & 0,18 & 10 & 0,05 & 47 & 0,23 \\
\hline L. flaviscutellata & 129 & 0,65 & 720 & 3,63 & 849 & 4,29 \\
\hline L. fischeri & 661 & 3,34 & 430 & 2,18 & 1.091 & 5,51 \\
\hline L. furcata & 17 & 0,08 & 9 & 0,04 & 26 & 0,18 \\
\hline L. hermanlenti & 12 & 0,06 & 23 & 0,11 & 35 & 0,17 \\
\hline L. infraspinosa & 112 & 0,56 & 61 & 0,28 & 173 & 0,84 \\
\hline L. lenti & 45 & 0,22 & 26 & 0,13 & 71 & 0,35 \\
\hline L. longipalpis & 775 & 3,91 & 529 & 2,57 & 1.304 & 6,48 \\
\hline L. longipennis & 321 & 1,62 & 71 & 0,35 & 392 & 1,97 \\
\hline L. lutziana & 75 & 0,37 & 49 & 0,25 & 124 & 0,62 \\
\hline L. monstruosa & 21 & 0,10 & 7 & 0,03 & 28 & 0,13 \\
\hline L. nevesi & 117 & 0,59 & 166 & 0,84 & 283 & 1,43 \\
\hline L. oliveirai & 96 & 0,48 & 38 & 0,20 & 134 & 0,68 \\
\hline L. peresi & 9 & 0,04 & - & . & 09 & 0,04 \\
\hline L. punctigeniculata & 217 & 1,09 & 135 & 0,70 & 352 & 1,77 \\
\hline L. quinquefer & 196 & 0,99 & 72 & 0,36 & 268 & 1,35 \\
\hline L. sallesi & 31 & 0,15 & 18 & 0,10 & 49 & 0,25 \\
\hline L. saulensis & 177 & 0,89 & 158 & 0,79 & 335 & 1,68 \\
\hline L. serrana & 216 & 1,09 & 146 & 0,74 & 362 & 1,83 \\
\hline L. shannoni & 315 & 1,59 & 976 & 4,93 & 1.291 & 6,52 \\
\hline L. sordelli & 6 & 0,03 & - & - & 08 & 0,04 \\
\hline L. damascenoi & 21 & 0,10 & 7 & 0,03 & 28 & 0,14 \\
\hline L. teratodes & 99 & 0,50 & 115 & 0,54 & 214 & 1,04 \\
\hline L. trinidadensis & 131 & 0,66 & 111 & 0,53 & 242 & 1,24 \\
\hline L. whitmani & 1.198 & 6,05 & 878 & 4,34 & 2.076 & 10,39 \\
\hline L. $s p$ & 0 & - & 59 & 0,30 & 59 & 0,30 \\
\hline Total & 10.636 & 53,62 & 9.154 & 46,38 & 19.790 & 100 \\
\hline
\end{tabular}


Tabela 2. Números e frequência de espécies de flebotomineos por estratégias de coletas, capturados no Municipio de Chapada dos Guimarães no periodo de Janeiro de 1993 a Dezembro de 1995.

\begin{tabular}{|c|c|c|c|c|c|c|c|c|c|c|}
\hline \multirow{2}{*}{ ESPÉCIES } & \multicolumn{2}{|c|}{$\begin{array}{c}\text { BURACOS, TOCAS } \\
\text { E PEDRAS }\end{array}$} & \multicolumn{2}{|c|}{$\begin{array}{l}\text { ARMADILHAS } \\
\text { SHANNON }\end{array}$} & \multicolumn{2}{|c|}{$\begin{array}{l}\text { ARMADILHAS } \\
\text { DISNEY }\end{array}$} & \multicolumn{2}{|c|}{$\begin{array}{c}\text { TRONCOS DE } \\
\text { ÄRVORES }\end{array}$} & \multicolumn{2}{|c|}{ CDC } \\
\hline & $\mathrm{N}^{2}$ & $\%$ & $\mathrm{~N}^{2}$ & $\%$ & $\mathrm{~N}^{2}$ & $\%$ & $N^{2}$ & $\%$ & $N^{2}$ & $\%$ \\
\hline B. avellari & 322 & 14,66 & 0 & - & 0 & - & 0 & - & 0 & - \\
\hline B. brumpti & 11 & 0,50 & 0 & - & 0 & - & 0 & - & 0 & - \\
\hline B. sp. & 7 & 0,31 & 0 & - & 0 & - & 0 & - & 463 & 46.21 \\
\hline L. abonnenci & 0 & - & 873 & 6,07 & 0 & - & 0 & - & 129 & 12,87 \\
\hline L. acanthopharynx & 0 & - & 0 & - & 0 & - & 0 & - & 0 & - \\
\hline L. antunesi & 0 & - & 289 & 2,01 & 0 & - & 0 & - & 0 & - \\
\hline L. aragoi & 0 & $\cdot$ & 268 & 1,86 & 0 & - & 0 & - & 0 & - \\
\hline L. ayrozai & 0 & - & 350 & 2,43 & 0 & - & 0 & - & 0 & $\therefore$ \\
\hline L. baity & 4 & 0,18 & 0 & - & 0 & - & 0 & - & 0 & - \\
\hline L. barretoi barretoi & 10 & 0,45 & 0 & - & 0 & . & 0 & - & 0 & . \\
\hline L. bourrouli & 0 & - & 0 & $\cdot$ & 0 & . & 8 & 0,46 & 0 & - \\
\hline L. brasiliensis & 48 & 2,18 & 0 & - & 0 & - & 0 & - & 0 & - \\
\hline L. campbeli & 8 & 0,36 & 0 & - & 0 & - & 0 & - & 0 & - \\
\hline L. carmelinoi & 23 & 1,04 & 0 & - & 0 & - & 0 & - & 0 & $\cdot$ \\
\hline L. carrerai carrerai & 0 & - & 344 & 2,40 & 0 & - & 0 & - & 0 & $\cdot$ \\
\hline L. claustrei & 0 & - & 78 & 0,54 & 0 & - & 0 & - & 0 & - \\
\hline L. complexa & 0 & - & 815 & 5,68 & 29 & 5,44 & 818 & 48,36 & 0 & - \\
\hline L. coutinhoi & 0 & - & 0 & - & 0 & - & 15 & 0,88 & 0 & - \\
\hline L. cruzi & 0 & - & 1851 & 12,88 & 0 & - & 0 & - & 0 & - \\
\hline L. davisi & 0 & - & 1612 & 11,22 & 0 & - & 0 & - & 0 & - \\
\hline L. dispar & 0 & - & 1301 & 9,06 & 164 & 30,77 & 0 & - & 0 & - \\
\hline L. evandroi & 37 & 1,68 & 0 & - & 101 & 18,95 & 0 & - & 0 & - \\
\hline L. flaviscuttellata & 0 & - & 620 & 4,31 & 10 & 1,88 & 0 & - & 0 & - \\
\hline L. fischeri & 0 & - & 1091 & 7,59 & 229 & 48,96 & 0 & - & 0 & - \\
\hline L. furcata & 26 & 1,18 & 0 & - & 0 & - & 0 & - & 0 & - \\
\hline L. hermanlenti & 35 & 1.59 & 0 & - & 0 & - & 0 & $\cdot$ & 0 & - \\
\hline L. infraspinosa & 0 & - & 0 & - & 0 & - & 173 & 10,22 & 0 & - \\
\hline L. lenti & 71 & 3,23 & 0 & - & 0 & - & 0 & - & 0 & - \\
\hline L. Iongipalpis & 588 & 26,77 & 716 & 4.99 & 0 & - & 0 & . & 0 & - \\
\hline L. Iongipennis & 392 & 17,87 & 0 & - & 0 & - & 0 & - & 0 & - \\
\hline L. lutziana & 124 & 5,64 & 0 & . & 0 & - & 0 & - & 0 & $\cdot$ \\
\hline L. monstruosa & 0 & - & 28 & 0,20 & 0 & - & 0 & - & 0 & . \\
\hline L. nevesi & 0 & - & 0 & - & 0 & - & 283 & 16,73 & 0 & - \\
\hline L. oliveirai & 0 & - & 134 & 0,94 & 0 & - & 0 & - & 0 & - \\
\hline L. peresi & 0 & - & 0 & - & 0 & - & 0 & - & 9 & 0,90 \\
\hline L. punctigenicutelata & 0 & - & 0 & - & 0 & - & 0 & . & 352 & 35,13 \\
\hline L. Quinquefer & 0 & - & 268 & 1,86 & 0 & - & 0 & - & 0 & - \\
\hline L. sallesi & 0 & - & 0 & - & 0 & - & 0 & - & 49 & 4,89 \\
\hline L. saulensis & 0 & - & 0 & . & 0 & - & 335 & 19,86 & 0 & - \\
\hline L. serrana & 0 & $\cdot$ & 362 & 2,52 & 0 & $\cdot$ & 0 & - & 0 & - \\
\hline L. shannoni & 0 & - & 1291 & 8,99 & 0 & - & 0 & - & 0 & - \\
\hline L. sordelli & 6 & 0,27 & 0 & - & 0 & - & 0 & $\cdot$ & 0 & - \\
\hline L. damascenoi & 28 & 1,27 & 0 & - & 0 & - & 0 & - & 0 & - \\
\hline L. Teratodes & 214 & 9,74 & 0 & - & 0 & - & 0 & - & 0 & - \\
\hline L. trinidadensis & 242 & 11,02 & 0 & - & 0 & - & 0 & - & 0 & - \\
\hline L. whitimani & 0 & + & 2076 & 14,45 & 0 & - & 0 & - & 0 & - \\
\hline L. sp. & 0 & - & 0 & - & 0 & - & 59 & 3,49 & 0 & - \\
\hline TOTAL & 2196 & 100 & 14367 & 100 & 533 & 100 & 1691 & 100 & 1002 & 100 \\
\hline
\end{tabular}


flebotômica dominante naquela região de alta endemicidade de leishmaniose tegumentar, era constituida principalmente pelas espécies; $L$. whitmani, L. pessoai, L. migonei; já em locais de baixa endemicidade para a leishmaniose tegumentar e fora das áreas florestais, L.. fischeri apareceu sempre como espécie dominante.

Em observações sobre criadouros e abrigos de flebotomíneos no Estado do Ceará, Deane \& Deane (1957) coletaram em troncos de árvores,espécimes das espécies $L$. abonnenci, L. longipalpis, L. oswaldoi, $L$. rickardi, $L$. trinidadensis e $L$. whitmani.

Damasceno \& Arouck (1949) em estudos realizados em diversas localidades da região Amazônica (Amazonas e Pará) capturaram 48 espécies de flebotomíneos com prodominância de $L$. dendrophilla, $L$. furcata e $L$. damascenoi. Das espécies por nós coletadas apenas $L$. brasiliensis, $L$. coutinhoi, $L$. infraspinosa e L. saulensis foram comuns às espécies coletadas por Damasceno \& Arouck (1949).

Vários autores realizaram coletas com a mesma estratégia na Reserva Florestal Ducke, Estado do Amazonas: Arias \& Freitas (1978); Ready et al. (1986); Silva (1994) sendo a espécie L. umbratilis a encontrada com maior frequência nesse tipo de habitat.

Aguiar \& Vilela (1987) em estudo sobre os aspectos ecológicos dos flebotomíneos no Estado do Rio de Janeiro, coletaram cinco espécies: L.. edwardsi, L.. monticola, L. pessoai, Lutzomyia sp. e L. fischeri, onde esta última mostrou predominância sobre as outras.

No estado de Roraima, Castellón et al. (1989) relataram a presença de 14 espécies, sendo L. ubiquitalis a de maior frequência, seguida de $L$. umbratilis.

Castellón et al. (1994) em trabalhos realizados na Rodovia Manaus-Humaitá, Estado do Amazonas, coletaram em maior número a espécie $L$. antunesi, seguida por L. umbratilis.

As evidências demonstradas pelos resultados de trabalhos em coletas nas bases de árvores, nas diferentes regiões do Brasil, indicaram uma diferença com as coletas realizadas na Chapada dos Guimarães, a exceção das quatro espécies obtidas por Damasceno \& Arouck (1949). Esta diferença pode ser atribuida aos diferentes tipos de vegetação e clima, inerentes a cada região e principalmente à capacidade de adaptação das diferentes espécies na busca de abrigos naturais.

\section{Coletas em Armadilhas Shannon}

O maior número de espécies (19) em nossos resultados, foram obtidos com esta estratégia (Tab. 2).

Forattini $(1954 ; 1960 b)$ e Foratini \& Santos (1955) coletaram flebotomíneos em várias localidades do Estado de São Paulo, utilizando armadilhas Shannon com iscas luminosas. Encontraram L. whitmani e L. intermedia com maior frequência nas coletas, seguidas de $L$. fischeri L. pessoai, L. shannoni, B. brumpti, B. avellari, L. monticola e L. arthuri, em 
menor número.

Forattinni \& Santos (1955) sugeriram: "Talvez estejamos assistindo a ocorrência de variações periódicas faunisticas devido a fatores ainda desconhecidos".

Forattini (1960a) concluiu que "as variações mensais dos flebotomíneos são influenciadas pelas condições climáticas, sendo maior a densidade desses insetos nas épocas quentes e úmidas e menor nas frias e secas". Concluiu ainda que "a composição especifica da fauna flebotômica sofre alterações com o passar do tempo, existindo certa alternância quanto á espécie dominante".

Vexenat et al. (1986) estudaram a fauna flebotômica na região de Três Braços, no Estado da Bahia, usando tambén armadilhas Shannon. Nessa área (região montanhosa que se liga à Mata Atlântica) coletaram 21 espécies com predominância de $L$. ayrozai, $L$. yuilli e $L$. fischeri. As espécies $L$. flaviscutellata, L. pessoai e L. whitmani tiveram poucos espécimes coletados na área de mata; no entanto de L. whitmani obteve-se em predominância em coletas no peridomicilio.

Das espécies coletadas na Chapada dos Guimarães, oito espécies foram comuns ás obtidas por Vexenat et al. (1986): L. aragaoi, L. carrerai, L. davisi, L. flaviscutellata, L. fischeri, L. shannoni e L. whitmani.

Theodoro et al. (1993) trabalharam em localidades de mata residual alterada no norte do Estado do Paraná, numa área de transmissão de leishmaniose tegumentar americana e capturaram dezesseis espécies de flebotomíneos com predominância de $L$. whitmani seguida de $L$. migonei $e$ $L$. intermedia alternadamente, e $L$. fischeri em menor frequência. Galati et al. (1996) desenvolveram estudos em Mato Grosso do Sul e revelaram que L. whitmani foi a espécie mais abundante com aproximadamente $96 \%$ do total.

Resultados quase semelhantes foram encontrados por Gomes \& Galati (1977) no Paraná; Gomes et al. (1978) no Estado de São Paulo; Magalhães (1977) em Minas Gerais; Rangel et al. (1986) em Rio de Janeiro, e Barros et al. (1985) no Espirito Santo.

\section{Coletas com Armadilhas Luminosas Tipo CDC}

O objetivo básico com este tipo de estratégia foi o de determinar qualitativa e quantitativamente a composição faunistica de flebotomíneos em diferentes estratos por altura.

Em geral, os nossos resultados demonstraram que houve uma diferença relativamente grande quanto à na diversidade e frequência das espécies coletados na área de Chapada dos Guimarães, quando comparados com os outros métodos de captura utilizados na área ou com a mesma estratégia usada em outras localidades do Brasil.

$\mathrm{Na}$ região de Chapada dos Guimarães, com o uso de CDC, as espécies de maiores frequências fo- 
ram: $L$. acanthopharynx seguida de $L$. antunesi, $L$. sallesi e $L$. peresi. Esses resultados indicam que a diversidade de espécies, atraidas por esta estratégia, foi baixa na área em estudo.

Arias \& Freitas (1982a; 1982b); Castellón et al. (1989; 1991a;1991b, 1994); Silva (1994) trabalharam em diferentes localidades da Amazônia utilizando esta estratégia de coleta e capturaram uma ampla diversidade de espécies.

Rangel et al. (1990) no Estado do Rio de Janeiro realizaram capturas conseguindo apenas duas espécies: $L$. intermedia e L. migonei.

Azevedo \& Rangel (1991) utilizaram CDC no Estado do Ceará e obtiveram quatro espécies diferentes: L. migonei, L. whitmani, L. shannoni e L. longipalpis.

Um número de fatores podem influenciar no rendimento quantitativo e qualitativo das espécies coletadas em diferentes localidades. Arias \& Freitas (1977); Shaw \& Lainson (1972) e Williams (1965) afirmaram: "diferentes tipos de capturas produzem resultados diferentes; o único método seguro para comparar tendências populacionais é usar a mesma metodologia. Além disso, as variações populacionais vão aumentar ou diminuir de acordo com outros fatores tais como pluviosidade, umidade do solo, alturas diferentes e iscas diferentes". De acordo com nossas observações, além dos fatores já mencionados, outros, tipicamente caracteristicos de cada região, podem ter influênciado. No caso de Chapada dos Guimarães as árvores são de pequeno porte, espaçadas e com penetração da luz solar; vegetação essa que não favoreceu como local de refugio adequado como no caso da região Amazônica. Além disso, a área é rupestre, bastante pedregosa com tocas, fendas e rachaduras que facilitariam o esconderijo de animais silvestres, possiveis fontes de hematofagia e que poderiam competir como área de refúgio, mais eficaz do que a vegetação.

\section{Coletas com Armadilhas Disney.}

Quatro espécies foram coletadas com esta estratégia, sendo: $L$, flaviscutellata a predominante, seguida de L. davisi, L.dispar e L. claustrei.

Shaw \& Lainson (1972); Ward et al. (1973); Ready et al. (1986) e Lainson et al. (1992) utilizaram essa metodologia em coletas seletivas no Estado do Pará, usando como isca os tambén roedores Oryzomys capito, Proechimys guyanensis elou edentados como Choloepus didactylus e Tamandua tetradactyla. Nos resultados, L. flaviscutellata, foi sempre a espécie dominante; segundo Lainson \& Shaw (1968) esta espécie é atraida "alimentando-se" de uma ampla variedade de animais silvestres; $L$.. flaviscutellata, na região amazônica, encontra-se adaptada na floresta de terra firme, preferivelmente associada a animais silvestres; é bem provável que esse tipo de comportamento venha ocorrendo na Chapada dos Guimarães. 


\section{Coletas em Buracos, Tocas e Pedreiras}

Tradicionalmente a estratégia mais utilizada tem sido o uso da armadilha de Damasceno. No nosso trabalho usamos a fumaça de cigarro dirigida aos buracos, tocas, frestas, e uma vez no exterior, os espécimes eram coletados com o capturador de Castro.

Nos Estados do Amazonas e Pará, Damasceno \& Arouck (1949) estudaram a distribuição geográfica e a incidência de captura das espécies de flebotomíneos, resultando na coleta de 48 espécies localizadas em tocas de animais silvestres no solo; $L$. dasipodogeton foi a espécie de maior frequêcia seguida de $L$. aragaoi, $L$. deanei e L. ubiquitalis.

No nordeste brasileiro, Deane \& Deane (1955) em observações sobre abrigos e criadouros no Ceará, coletaram espécimes nas fenda em rochas, grutas e sob pedras: $L$. longipalpis foi nitidamente predominante, seguida de $L$. oswaldoi, $L$. rickardi, $L$. shannoni, $L$. trinidadensis, L. whitmani e $L$. flaviscutellata. Guitton \& Sherlock (1969) na Bahia, também encontraram a predominância de $L$. longipalpis no interior de grutas.

Aguiar \& Vilela (1987) no Rio de Janeiro, coletaram em tocas de animais silvestres oito espécies: quatro de Brumptomyia: B. cardosoi, B. troglodytes, B sp . e B. guimaraesi e quatro de Lutzomyia: $L$. barrettoi, $L$. aragaoi, L. monticola e L. microps; no entanto, em fendas em pedras somente foi coletada L. hirsuta.

Das espécies por nós coletadas, L. longipalpis foi a mais frequente, dado este, semelhante aos resultados de Deane \& Deane (1957) e Guitton \& Sherlock (1969); demostrando a sua predominância neste tipo de habitat.

Três espécies de Brumptomyia : B. avellari, B. brumpti e B. sp. foram coletadas tal como nas coletas de Aguiar \& Villela (1987). Isto evidencia que as espécies desse gênero presentes em áreas com vegetação escassa, procuram abrigo neste tipo de topografia, o que difere de outras regiões como na Amazônia onde tais espécies podem ser encontradas preferencialmente em base de grandes árvores e tocas de animais silvestres.

\section{Bibliografia citada}

Aguiar, G. M.; Vilela, M.L. 1987. Aspects of the ecology of sandflies at the Serra dos Órgãos National Park, State of Rio de Janeiro. VI. Shelters and breeding places (Diptera: Psychodidae, Phlebotominae). Mem. Inst. Oswaldo Cruz, 82: 488-584.

Arias, J. R.; Freitas, R. A, 1977. On the vector of cutaneous leishmaniasis in the Central Amazon of Brazil. I. Preliminary findings. Acta Amazonica 7:293-294.

Arias, J. R.; Freitas, R. A. 1978. Sobre os vetores de leishmaniose cutânea na Amazônia do Brasil. 2: Incidência de flagelados em flebótomos selváticos. Acta Amazonica, 8:387-396.

Arias, J. R.; Freitas, R. A. 1982a. The known geographical distibution of sand flies in the state of Acre, Brazil (Diptera: Psychodidae). Acta Amazonica, 12(2):401408 .

Arias, J. R.; Freitas, R. A. 1982b. On the vectors of cutaneous leshmaniasis in the Cen- 
tral Amazon of Brazil. 3. Phletomine sandfly stratification in a terra firme forest. Acta Amazonica, 12(3):599-608.

Azevedo, A. C. R.; Rangel, E.F. 1991. A study of sandfly species (Diptera: Psychodidae, Phlebotominae) in a focus of cutaneous leishmaniasis in the municipality of Baturité, Ceará, Brazil. Mem. Inst. Oswaldo Cruz, 86(4):405-410.

Barros, G. C.; Sessa, P. A; Matos, E. A; Carias, V. R. D; Mayrink, W.; Alencar, T. A.; Falqueto, A.; Jesus, A.C. 1985. Focus of mucocutaneous leishmaniasis in Viana and Cariacea counties, Espirito Santo state, Brazil. Rev. Saúde Publica., 19:146-153.

Castellón, E. G.; Araújo Filho, N. A.; Fé, N. F.; Alves, J. M. C. 1989. Flebotomineos (Diptera:Psychodidae) no Estado de Roraima, Brasil. I. Espécies coletadas na Região Sul e Central. Mem. Inst. Oswaldo Cruz, 84(4):95-99.

Castellón, E.G.; Araújo Filho, N.A.; Fé, N.F.; Alves, J.M.C, 1991a. Flebotomineos (Diptera:Psychodidae) no Estado de Roraima. Il. Espécies coletadas na Região Norte. Acta Amazonica, 21:45-50.

Castellón, E.G.; Araujo Filho, N. A.; Fé, N.F.; Alves, J.M.C. 1991b. Flebotomineos coletados no Estado de Roraima, Brasil. III. Listagem das espécies, coletadas na Estação Ecológica na Ilha de Maracá e no Estado. Acta Amazonica, 21:51-54.

Castellón, E.G.; Arias, J.R; Freitas, R.A.; Naiff, R.D. 1994. Os flebotomineos da região Amazônica, estrada ManausHumaitá, Estado do Amazonas, Brasil (Diptera: Psychodidae: Phlebotominae). Acta Amazonica, 24 (1/2):91-102.

Christensen, H.A.; Vasquez, A.M. 1982. The tree-bruttress Biotope: a pathobiocenose of Leishmania braziliensis. Am. J. Trop. Med. Hyg., 31(2) : 243-25I.

Coutinho, J.O.; Barretto, M.P. 1941. Dados bionômicos sobre o Phlebotomus fischeri, Pinto, 1926 (Diptera:Psychodidae). Revta. brasil. Biol.,1(4):422-310.

Damasceno, R.G.; Arouck, R. 1949. Estudos sobre Flebótomus no Vale Amazônico.
Parte VII. Descrição de uma espécie nova. Revta. Serv. Espc. Saúde. Pública, 2(3): 843-848.

Deane, L.M.; Deane, M.P. 1955. Observações sobre a transmissão de leishmaniose visceral no Ceará. Hospital, Rio de Janeiro, 48(3):347-364.

Deane, L.M.; Deane, M.P. 1957. Observações sobre abrigos e criadouros de flebótomos no Nordeste do Estado do Ceará. Revta. brasil. Malar. Doenç. Trop., 9(2): 225 246.

Forattini, O.P. 1954. O segundo esternito em algumas espécies de flebótomos do Brasil (Diptera, Psychodidae). Folia Clin. Biol., 21:213-218.

Forattini, O.P. 1960a. Novas observações sobre a biologia de flebótomos em condições naturais (Diptera, Psychodidae). Arq. Hig. São Paulo, 25:209-215.

Forattini, O.P. 1960b. Sobre os reservatórios naturais para Leishmania Tegumentar Americana. Revta. Inst. Med. Trop. São Paulo, 2(1):195-203.

Forattini, O.P.; Santos, M.R. 1955. Novas observaçōes em regiōes endêmicas de leishmaniose tegumentar Americana nos estados de São Paulo e Mato Grosso, Brasil. Revta. Clin. São Paulo, 1-3:13-20.

Fraiha, H.; Ward, R.D.; Shaw, J.J; Lainson, R. 1978. Fauna antropófila de flebótomos da rodovia Transamazônica, Brasil (Diptera, Psychodidae). Bull. Pan . Amer. Health Org., 84: $143-139$.

Fundação Cândido Rondon. 1983. Municipio de Mato Grosso - Chapada dos Guimarães. 125p.

Galati, E.A.B.; Nunes, V.L.B.; Dorval, M.E.C.; Oshiro, E.T.; Cristaldo, G.; Espidola, M.A.; Rocha, H.C.; Garcia, W.B. 1996. Estudo dos flebotomineos (Diptera: Psychodidae), em área de leishmaniose tegumentar, no Estado de Mato Grosso do sul, Brasil. Rev. Saúde Pública, 30 (2): $115-28$.

Gomes, A.C.; Galati, E.A.B. 1977. Flebotomíneos de Londrina, Paraná, Brasil e observaçòes ecológicas sobre 
algumas espécies. Rev. Saúde Pública, 11: 284-287.

Gomes, A.C.; Rabelo, E.X.; Galati, E.A.B. 1978. Flebotomineos encontrados em galinheiros experimentais nos estados de São Paulo e Minas Gerais, (Brasil) e algumas observações ecológicas. Rev. Saúde., Pública., 12: 403-407.

Guitton, N.; Sherlock, I.A, 1969. Descrição das fases imaturas do Lutzomyia longipalpis (Lutz \& Neiva, 1912) (Diptera, Psychodidae). Revta. Brasil. Biol., 29: 383-389.

Lainson, R. 1983. The american Leishmaniasis. Some obervations on their ecology and epidemiology. Trans. R. Soc. trop. Med. Hyg., 77(5):569-596.

Lainson, R.; Shaw, J.J. 1968. Leishmaniasis in Brasil. I. Observations on enzootic rodent leishmaniasis - incrimination of Lutzomyia flaviscutellata (Mangabeira) as the vector in the lower amazonian basin. Trans. $R$. Soc. trop. Med. Hyg., 62(3): 385-395.

Lainson, R.; Shaw, J.J. 1989, Leishmania (Viannia) naiffi $\mathrm{sp} . \mathrm{n}$. a parasite of the armadillo, Dasypus novemcintus (L.) in Amazonian Brazil. Ann Parasitol. Hum. Comp., 64 (1) : 3-9.

Lainson, R.; Shaw J.J.; Souza, A.A.A.; Silvera F.T.; Falgueto, A. 1992. Further observations on Lutzomyia ubiquitalis (Psychodidae : Phlebotominae), the sandfly vetor of Leishmania (Viannia) lainsoni. Mem. Inst.Oswaldo Cruz, 87(8): 437-439.

Magalhães, R.A. 1977. Leishmaniose no Vale do Rio Doce. Anais Bras. Derm. Sif., 52(3): 319-324.

Rangel, E.F.; Souza, N.A.; Wernelinger, E.D.; Azevedo, A.C.; Barbosa, A.F.; Andrade, C. A. 1986. Flebótomos de Vargem Grande, foco de leishmaniose tegumentar no Estado do Rio de Janeiro. Mem. Inst. Oswaldo Cruz, 81(3) : 347-349.

Rangel, E.F.; Azevedo, A.C.R.; Andrade C.A.; Souza, N.A.; Wermelinger, E.D.; 1990. Studies on sandfly fauna (Diptera: Psychodidae) in foci of cutaneous leishmaniasis in Mesquita, Rio de Janeiro state,
Brasil. Mem. Inst. Oswaldo Cruz, 85 (1) : $39-45$

Ready, P.D,; Lainson, R.; Shaw, J.J.; Ward, R.D. 1986. The ecology of Lutzomyia umbratilis Ward \& Fraiha (Diptera : Psychodidae), the major vector to man of Leishmania braziliensis guyanensis in north eastem Amazonian Brazil. Bull. Ent. Res., 76: 21-40.

Shaw, J.J.; Lainson, R. 1972. Leishmaniasis in Brasil. VI. Observation on the seasonal variations of Lutzomyia flaviscutellata in different types of forest and its relationship to enzootic rodent leishmaniasis (Leishmania mexicana amazonensis). Trans. R. Soc. Trop. Med. Hyg., 66: 709-717.

Silva, M.R.C. 1994. Estudo sobre abrigos naturais dos Phlebotominae (Diptera : Psychodidae) na Reserva Florestal Adolfo Ducke, Manaus-AM, Brasil. Dissertação de Mestrado. INPA / UFAM. Manaus. $105 \mathrm{p}$.

Teodoro, U.; Filho, V.S.; Lilma, E.M.; Spinosa, R.P.; Barbosa, O.C; Ferreira, E.M.C.; Lonardoni, M.V.C. 1993. Observações sobre o comportamento de flebotomineos em ecótopos florestais e extraflorestais, em área endêmica de leishmaniose tegumentar americana, no Norte do Estado do Paraná, Sul do Brasil. Rev. Saúde Pública., 27(4): 242-249.

Vexenat, J.A.; Barreto, A.C.; Cuba, C.; Marsden, P.D. 1986.Caracteristicas epidemiológicas da Leishmaniose tegumentar americana em uma região endêmica do Estado da Bahia. III. Fauna flebotômica. Mem. Inst. Oswaldo Cruz, 81: 293-301

Ward, R.D.; Shaw, J.J.; Lainson, R. 1973. Leishmaniasis in Brasil. VIII. Observations on the phlebotomine fauna of an area highly endemic for cutaneous leishmaniais, in the Serra dos Carajás, Pará state. Trans. R. Soc. Trop. Med. Hyg., 67: 174-183.

Williams, P. 1965. Observations on the phlebotomine sandflies of British Hondu- 
ras. Ann. Trop. Med \& Parasitol., 59(4); 373-404.

Young, D.G.; Arias, J.R. 1991. Phlebotomine Sandflies in the Americas. Pan American Health Organization. Pan American Sanitary Bureau, Regional Office of the, World Health Organization, Technical Paper $\mathrm{n}^{0} 33$.

Young, D.G.; Duncan, M.A. 1994. Guide to the identification and geographic distribution of Lutzomyia sand flies in Mexico, the West Indies, Central and South America (Diptera: Psychodidade). Memoirs of the American Entomological Institude, $\mathrm{n}^{-}$54. Associated Publishers, Gainesville, Florida, USA, 881p. 